\title{
CARL BENJAMIN HERMANN BARON VON ROSENBERG.
}

\author{
EINE BIOGRAPHISCHE SKIZZE
}

V ON

Dr. Th. C. L. WIJNMalen.

In der Einleitung zu "v on Rosenberg: Der Malayische Archipel" schrieb Herr Professor P. J. Veth u. a. mit Recht:

"Sicherlich wird Niemand, der nur einigermassen bekannt ist mit dem Umfang und Werth desjenigen, was in den letzten fünfzig Jahren für die Kenntniss der Sprachen und Literatur von Niederländisch-Indiën von Niederländern geleistet ist, die Gebieter der indischen Inseln der Fahrlässigkeit und Gleichgültigkeit für wissenschaftliche Interessen zeihen; aber es kann nicht geleugnet werden, dass neben den hervorragenden Namen eines Roorda, Cohen Stuart, van der Tuuk, Matthes, Kern, Pijnappel und Anderer auf dem Gebiete der sprachlichen und literarischen Forschungen, die Niederländer in ihren eignen Kolonien auf dem der Naturwissenschaft etwas den Fremden nachstehen und nicht viele Namen aufzuweisen haben, die mit denen eines Reinwardt, Blume, Salomon Müller, Horner, Kuhl, Hasskarl, Junghuhn, Bernstein, Miquel, Hermann Schlegel, Emil Stöhr (alle geborene Deutsche, wenn auch zum Theil in Holland erzogen) in gleiche Linie gestellt werden können." "

Jenen letzten Namen darf jetzt auch der von Carl Benjamin Hermann von Rosenberg beigezählt werden. Auch er "gehört", allerdings, "zu der beträchtlichen Zahl derjenigen Deutschen, die im Dieuste der niederländischen Regierung und oft in ihrem speziellen Auftrag an der Untersuchung der malayischen Inselwelt lebhaften Antheil genommen. "

Einer alt-adeligen Hessischen Familie entsprossen - er sah am 7ten April 1817 in Darmstadt (Hessen) das Lebenslicht hatte von Rosenberg sich im Mai 1865 naturalisieren lassen (Siehe 
Gesetz von 27ten Mai 1865, Gesetz-sammlung $n^{r}$. 43); es kam dem Edelmann, in seinem ritterlichen Herzen, niemals in den Sinn das Land, wo seine Wiege stand, in welchem er seine Jugend und seine Jünglingsjahre verbracht hatte zu verläugnen; vielmehr rühmte er Land und Volk und blieb es ehren; nicht weniger anhänglich zeigte er sich jedoch gegen sein Adoptivvaterland, welches gleichfalls wiederum den Besitz dieses angenommenen Sohmes hochschätzte.

Aus einer Autobiographie, welche er uns hinterliess, so wie aus den verschiedenen Berichten seiner Reisen und Forschungen können wir die wichtigsten Begebenheiten aus seinem Leben kennen lernen.

Dem Wunsche seines Vaters 1 gemäss, war er anfänglich zum Militärdienste bestimmt und erhielt seine Ausbildung auf dem Gymnasium und der Kadetten-Schule in seiner Geburtsstadt. 1833 finden wir ihn als Kadett im Hessischen Militärdienste, aber unter den damaligen Verhältnissen (Anfang der 30er Jahre) muthete ihn dies wenig an und widmete er sich mehr naturwissenschaftlichen Studien. Dank dem Einfluss seines Oheims, erwachte schon früh in ihm eine lebendige Theilnahme für die Kenntniss fremder Iänder und Völker, und waren Reisebeschreibungen und naturhistorische. Werke seine Tieblingslektüre. Anfänglich beschäftigte er sich auf dem Polytechnikum in Carlsruhe mit naturwissenschaftlichen Studien; diese wurden nachher unter der Leitung seines späteren Freundes, Dr. J. J. Kaup in Darmstadt fortgesetzt, wobei das Studium der Zoologie in den Vordergrund trat. Seinen feurigen Wunsch, fremde Länder zu besuchen, besonders die Tropen kennen zu lernen, konnte er jedoch, unbemittelt wie er war, nicht befriedigen. Nach seiner Entlassung aus dem Hessischen Militärdienste, 1 April 1839, fasste er den kühnen Entschluss sich als Volontär bei der Niederländisch-Indischen Armee an zu melden; den 10ten Mai 1839 liess er sich für sechs Jahre zu Harderwijck anwerben, wurde am 1 sten September zum Korporal ernannt, und reiste den 23 November darauf, 22 Jahre alt, aus Hellevoetsluis nach Indien auf dem Schiffe "Batavia" ab, welches jedoch zuvor St. George d'Elmina, auf der Westküste Afrika's berühren musste, um afrikanische Rekruten aufzunehmen. Den 2ten Mai 1840 setzte von Rosenberg zum ersten Male den Fuss auf Java, jenes heiss ersehnte Ziel seiner Wünsche. Ein Monat später wurde er nach Padang, auf Sumatra's Westküste, versetzt, und bald sah er sich dem berühmten Geologen Dr. F. Junghuhn als Gehülfe bei-

\footnotetext{
1 Sein Vater, Herr Carl Ferdinand von Rosenberg, war Grossherz. Hess. Oberst.
} 
gefügt, womit die Periode seiner Reisen und Forschungen beginnt.

Von 1840 bis 1845 war von Rosenberg in einem Theil der Battaländer. Den 2ten Oktober $1840 \mathrm{kam}$ er mit der indischen Barke "Justina" in Begleitung Dr. Junghuhn's auf der Rhede von Tapanuli an. Nach den nöthigen vorbereitenden Massregeln wurde die Reise nach den Battaländern am 4ten Oktober angetreten, zuerst nach Lumut, wo von Rosenberg die Ankunft Junghuhn's, der einige Tage später abzureisen gesonnen war, abwarten sollte. Seinen Aufenthalt an diesem, mitten im Walde auf einer Anhöhe gelegenen Orte benutzte er zu Jagdstreifereien in der Umgegend, jedoch nicht ohne Gefahr für sein Leben. Nur von einem malayischen Bedienten, Hassan, begleitet, hatte er sich waldwärts begeben, um einen Vogel zu erlangen, dessen Laut er wahrzunehmen vermeinte und der sich als ein Phoenicophaeus erwies. Seine Mühe wurde nicht belohnt, im Gegentheil, er verlor den Weg und verirrte sich immermehr. Er selbst hat uns dieses Abenteuer geschildert, welches, wie er schreibt, mit unauslöschlichen Zügen seiner Erinnerung eingeprägt blieb, weil es leicht ein trauriges Ende hätte nehmen können, würde nicht das rechtzeitige Auftreten Junghuhn's der sich über das lange Ausbleiben von Rosenberg's beunruhigte, ihn aus seiner misslichen Lage gerettet haben. Neuling in den Tropen und wahrscheinlich in Folge dieses Ereignisses bekam von Rosenberg einen starken Fieberanfall, der bald zum Wechselfieber wurde und ihn so schwächte, das der Assistent die Begleitung Junghuhn's aufgeben und im November seine Entlassung nehmen musste. Von Rosenberg wurde alsdanu als Unterofficier der 7ten Compagnie der 7 ten Depot-Abtheilung zugetheilt, welche zu Pertibie, 5 Tagereisen weiter im Innern, garnisonierte. Dort verblieb er bis zur Aufhebung des Postens im April des Jahres 1843; die Zeit seines Aufenthalts daselbst wendete er hauptsächlich auch zur Aufnahme und Untersuchung der Ruinen von Hindutempeln bei Burumon an.

Am 14ten April 1843 wurde Pertibie auf Befehl der Regierung geräumt und den Truppen das Dorf Tobing als Garnisonsplatz angewiesen, für von Rosenberg ein entzückender Ort, weil derselbe äusserst günstig gelegen ist für einen Sammler naturhistorischer Gegenstände. In diesem Jäger-Eldorado blieb er bis zu seiner Versetzung nach Siboga am 19ten Oktober 1844. Während dieser Zeit nahm er auch an zwei Expeditionen Theil, die erste gegen die aufrührischen Batta-Dörfer Rapat und Parik-di-Batta vom 29 Januar bis zum 16 Februar 1844; die zweite vom 15 Juli bis 6 August, eigent- 
lich mehr ein militärischer Marsch nach dem Thal von Silindong.

Im Oktober nach Siboga versetzt, machte von Rosenberg im November an Bord der Kriegsbrigg "Haai" als Begleiter des Majors van der Hart eine Reise nach Baros-Singkel und Trumon, und erhielt er im Januar 1845 seine Versetzung zum Generalstab nach Padang, wohin er am 4, ten desselben Monats an Bord des Kriegsschooners "Argo" abreiste. Nach einer mehr als vierjährigen Abwesenheit kam er am $11^{\text {ten }}$ Januar wieder nach Padang zurück und blieb daselbst, mehrere später zu erwähnende Unterbrechungen abgerechnet, bis zum 29 Mai 1856.

Von Rosenberg wurde auf dem Stabs-Bureau als Zeichner in Thätigkeit gestellt, und dass er bei der Ausführung der ihm aufgetragenen Arbeiten sehr viel Fähigkeit und Eifer an den Tag gelegt hat, beweist das schmeichelhafte schriftliche Zeugniss das ihm durch den Kapitän-Chef des Stabes des Militärcommando's von Sumatra's Westküste zu Padang am 12ten Juli 1847 ḅehändigt wurde.

Vom 29 April bis 2 Juli 1845 wurde eine Reise nach der IX Kota unternommen; im Juni 1846 und im November und December 1849 eine Untersuchung der kleinen, nördlich der Rhede liegenden Inseln: Tudju Pulu (sieben Inseln); vom 24. Juni bis 28 Oktober 1849 Reise nach Agam und der IV Kota; vom 19 Mai bis 8 August 1852: Reise nach den östlichen Distrikten der Hochländer von Padang; nach den Mentawej-Inseln wurden drei Reisen gemacht: die erste, vom 24 März bis 9 Mai 1847; die zweite vom 24 März bis 9 Juni 1849; diedritte vom 27 August bis 8 Sept. 1852; darauf wurde die Insel Engano vom 10 bis 24 September desselben Jahres besucht. Von der Regierung mit einer geodätischen Aufnahme der Provinz Singkel und den dazu gehörigen Banjak-Inseln beauftragt, trat Rosenberg am 29 Januar 1853 die Reise dahin an. Sowohl die nächste Umgebung der Abtheilung Singkel als die angrenzenden Länder wurden aufgenommen, wonach die Untersuchung der Banjak-Inseln stattfand, welche am 12 Juli begann und bis zum 15 August 1853 dauerte.

Nach Singkel zurückgekehrt, verliess er seinen Aufenthaltsort bald wieder um die topographische Aufnahme des südlichen Theils der Provinz von dem dafür näher gelegenen Etablissement Baros aus zu bewerkstelligen. Erst Ende Oktober wurde dise Arbeit beendet und im December trat Rosenberg die Rückreise nach seinem Standort Padang, über Siboga und Natal an, wo er am 11 Januar 1854 eintraf. Im April wurde ihm die Terrain-Aufnahme zwischen Padang und der XIII Kota aufgetragen, die er bald erledigte indem kurz darauf 
(27 Mai bis 8 Juni 1854), gemäss einer Verabredung mit zwei in Soloh stationierten Freunden, der Vulkan Talang oder Sulassie erstiegen wurde.

Der Beamte Nieuwenhuizen, derzeitiger Chef der Provinz Singkel und von Rosenberg wurden als Commissäre für eine Untersuchung der Insel Nias ernannt, wobei Letzterem noch speziell die Aufnahme einer Karte anvertraut wurde. Der Aufenthalt auf dieser Insel dauerte vom 6 September 1854 bis 11 September 1855; bevor R. aber nach Padang zurückkehrte, zu welchem Zwecke er sich bereits zu Gunung-Sitoli eingeschifft hatte, wurde er während der Reise dahin durch schwere Winde und Stürme genöthigt Udjung (Kap) Tapak, die Südspitze von Simalu, zu besuchen, wodurch er eine Insel kennen lernte, die ein Jahr zuvor von dem damaligen Residenten Tapanuli's besucht wurde und der sicherlich der erste Europäer gewesen ist, dessen Fuss die Insel betrat. Bis den 22sten September blieb er auf dieser Insel, um dann die Rückreise nach Siboga und weiter nach Padang zu unternehmen, wo er am 7 Nov. ankam. Sein Aufenthalt auf Sumatra nahte bald dem Finde; vom 28 Febr. bis 25 März 1856 hielt er sich noch aus Gesundheitsrücksichten zu Brakian auf, in einer Gegend, welche mit Recht den Namen eines Eldorado, wenigstens für Ornithologen und Entomologen verdient. Im Mai desselben Jahres verliess er Sumatra's Westküste endlich, nachdem er daselbst mehr als 16 Jahre zugebracht hatte.

Mittlerweile war von Rosenberg, nachdem er schon 26 Oktober 1841 Furier geworden, zu dem Rang eines Adjutant-Unterofficier (Feldwebel) erhoben und wurde er im Juni 1856 in seiner Militärcharge dem Topographischen Bureau in Batavia attachirt. Zu Ende des Jahres 1857 hatte die Indische Regierung eine Commission ernannt für die Untersuchung und Feststellung eines Ortes auf Neu-Guinea, der zur Anlage einer Regierungsniederlassung geeignet wäre. Von der Regierung designiert die Reise dahin als Zeichner mitzumachen : schiffte er sich am 9ten Januar 1858 in Batavia an Bord des Postdampfers "Macassar" ein, um über Samarang, Surabaja, Macassar, Timor-Kupang und Banda nach Amboina zu reisen, woselbst er am 2ten Februar anlangte. Nachdem die Mitglieder der Commission, die sich an verschiedenen Orten der Kolonie befanden, endlich alle eingetroffen, bestiegen das Mitglied der Commission, Kapitän der Infanterie Beckmann, Lieutenant Tissot von Patot, Commandant eines Truppendetachements und von Rosenberg, die Barke "Atie-atul-barie und verliessen am darauffolgenden Tag Amboina, während der "Etna" 
auf welchem die übrigen Mitglieder der Commission sich eingeschifft hatten, 3 Tage später folgen sollte. Die Reise wurde nach der Südwest- und der Nordost-Küste Neu-Guinea's unternommen; die Untersuchung dieser Gegenden, der Aufenthalt an der Südwestküste, auf Dorej, und an der Humboldtbai dauerte vom 15 März bis 21 Juli 1858, worauf man, nach einer Abwesenheit von 137 Tagen, wieder nach Amboina zurùckkehrte, woselbst von Rosenberg einige Zeit verblieb.

Im folgenden Jahre, 21 Februar 1859, verliess er den Militärdienst, nachdem schon am 24 Mai $1853 \mathrm{ihm}$ die silberne Medaille zuerkannt war für 24, jährigen treuen Dienst. In den Civildienst der Regierung übergetreten, nachdem er das Examen für den hŏheren Beambtendienst abgelegt, wurde er zeitlich zur Verfügung des Gouverneurs der Molukken gestellt, und sah sich bald temporär beauftragt mit der Civilverwaltung der Südküste Seram's und speziell damit, eine Karte der Insel zu entwerfen. Durch grössere und kleinere Reisen, nun von ihm unternommen, hatte er Gelegenheit einen beträchtlichen Theil der Insel und deren Bewohner kennen zu lernen. Bei der Verlegung des Sitzes der Verwaltung vom Dorfe Paulohi nach Amahei wurde er beauftragt mit Benutzung der brauchbaren, zu Paulohi vorhandenen Materialien ein neues Fort zu erbauen. Kaum hatte er sich dieser Aufgabe entledigt, als er den Befehl erhielt eine Wohnung für einen Missionär zu bauen, welchem Amahei zum Standplatz angewiesen war. Er brachte auch die fertig, obschon er in seinem ganzen Leben niemals etwas gebaut; hätte er nachgesucht beider Aufträge enthoben zu werden, so würde die Regierung dies, und zwar seiner Meinung nach mit Grund, übel vermerkt haben, da sie verlangt, dass ihre Beamten, wenn nöthig, nach jeder Richtung hin brauchbar sein müssen. Das Eine und das Andere nahm indessen den grössten Theil seiner Zeit in Anspruch; im Oktober 1860 wurde er denn auch von der Civilverwaltung enthoben und ausschliesslich mit der geodätischen Aufnahme der Insel betraut.

Hintereinander wurden die Sülküste von Seram von der TolutiBay bis zur Ostspitze der Insel, die Seram-laut-Gruppe und Seram's Nordküste von Kap Musiha bis Wahai bereist; auch wurden Fussreisen unternommen quer durch Seram von der Süd- nach der Nordküste. Ueber zwei und ein halbes Jahr, vom 26 April 1859 bis 17 Dec. 1861, danerte der Aufenthalt auf dieser Insel, indem mittlerweile während der Monate Juli und August 1860 von dem auf Seram's Nordküste gelegenen Wahai aus eine Untersuchungsreise unternommen wurde nach den Papua-Inseln Misool, Batanta, Salawatti und Waigeü, 
Im Januar 1862 war er leider gezwungen seine Arbeiten zu beendigen und behufs Wiederherstellung seiner zerrütteten Gesundheit um einen Urlaub nach Java zu ersuchen; dieser wurde ihm mittelst Ind. Beschluss vom 25 April 1862, $\mathrm{n}^{\mathrm{r}} 10$ gestattet, und mittelst derer vom 7 Mai 1862, $\mathrm{n}^{\mathrm{r}} 46$ und 2 Juni 1862, $\mathrm{n}^{\mathrm{r}} 2$, wurde dieser Urlaub jedesmal mit einem Monat verlängert. Nach seiner Wiederherstellung wurde er am 4ten Juni des letztgenannten Jahres zum Beamten, beauftragt mit naturwissenschaftlichen Untersuchungen und dem Sammele von Gegenständen für das Zoologische Reichsmuseum zu Leiden ernannt. Als solcher machte er von seinem Standorte Amboina aus (April 1863-August 1864) Reisen nach der Abtheilung Gorontalo, der Tomini-Bay und den Togean-Inseln (Nord-Selebes); vom 6 Januar bis 5 Oktober 1865 fand die wissenschaftliche Untersuchung der Aru- und Süd-Oster Inseln statt, namentlich der KeiInseln, der Inseln Koor und Tijoor, der Watubella-Inseln und der Goram-Gruppe, indem auf der Durchreise die Insel Timor, die BandaGruppe, Boerve und Ternate besucht wurden.

In Folge dieser vielen Reisen hatte seine Gesundheit sehr gelitten und waren seine Leibeskräfte geschwächt, er sah sich daher gezwungen einen zweijährigen Urlaub nach Europa zu nehmen, welcher ihm mittelst Ind. Beschluss von 6 Januar $1866 \mathrm{n}^{\mathrm{r}}$. 3 zugestanden wurden. Von Rosenberg suchte und fand die Wiederherstellung seiner erschütterten Gesundheit in seinem Geburtslande, zu Darmstadt.

Auf einer kleinen Reise durch Süddeutschland die unser Freund, Herr Schmeltz, zu jener Zeit von der Naturforscher Versammlung in Frankfurt a. M. aus unternahm, hatte er Gelegenheit in einem Kreise Gleichgesinnter von Rosenberg in Darmstadt kennen zu lernen. Mit grossem Enthusiasmus sprach er derzeit von seinem Aufenthalt in Indien, von neuen Plänen und neuem Schaffen, gleicḥ einem zwanzigjährigen Jüngling. Nie hätte Schmeltz geahnt, als Conservator am Leidener Ethnographischen Reichs-Museum, von Rosenberg nach langen Jahren in Niederland persönlich wieder zu begegnen und manch freundliches, ermuthigendes Wort von ihm noch zu hören; gerade wie an jenem Abend war von Rosenberg derselbe geblieben.

Nach Ablauf seines Urlaub's kehrte von Rosenberg nach Indiën zurück, wo er mittelst Ind. Beschluss von 15 Juni $1868 \mathrm{nr}$. 4 nochmals mit wissenschaftlichen Untersuchungen beauftragt wurde, unter Erlaubniss, wenn nöthig, auf seinen Reisen das Amtskleid eines Assistent-Residenten zu tragen. Nochmals ging er nach den Molukken; den $6^{\text {ten }}$ August 1868 von Batavia abgereist, erreichte er am 4 Sep- 
tember Ternate, den Ausgangspunkt seiner Untersuchungsreisen nach Neu-Guinea 1. Die Monate Oktober, November und December verflosen unter Zurüstungen für diese Reisen. Die Mussestunden, welche ihm wïhrenddem übrigblieben, benutzte er zu Ausfligen auf der Insel selbst und nach Tidore und Halmaheira, indem seine Untersuchungen daselbst später, 9 Juli 1869 bis 10 Januar 1870,10 Juni 1870 bis 26 Mai 1871 , wiederholt und ausgebreitet wurden. Vom $16 \mathrm{De}-$ cember 1868 bis Juli 1869 finden wir ihn auf Reisen im GeelvinkBusen; 4 Januar 1870 ging er nochmals dahin, uber Batjan nach Andai, um im folgenden Juni von da nach Temate zuridck zu kehren, woselbst er bis zur Mitte des Jahres 1871 blieb. Er fuhlte sich nicht mehr kräftig genug um seine Dienstgeschafte nach Pflicht und Gewissen $z$ verrichten. Er erbat sich laut und erhelt daher Ind. Beschluss vom 7 September $1871 \mathrm{n}^{\mathrm{x}}$. 54 eine ehrenvolle Entlassung aus dem Landesdienste, mit Zuerkennung siner Jahrespension von fl. 2250 (Beschluss von 2 Oktober $1871 \mathrm{nn}^{\mathrm{t}}$, 5). Noeh in demselben Jahre reiste er über Aegypten nach Europa ah. "Schwer", schreibt er, "fiel mir der Abschied von dem Lande der Sonne und des Lichtes; ob ich es wohl jemals wiedersehen werde?" Er machte 1876 einen Versuch dazu, aber sein Ansuchen um auf's Neue zum wissenschaftlichen Beamten ernannt zu werden, wurde mit Ind. Beschluss von 6 Mai $1876 \mathrm{nr}$. 32 abgewiesen.

Die Früchte der vieljährigen Untersuchungen und Reisen von Rosenberg's wurden in mehr als einer Schrift, in mehr als einem Aufsatz niedergelegt, meistentheils von wissenschaftlichen Stiftungen herausgegeben.

Die Zeitschrift des Bataviaschen Vereins der Künste und Wissenschaften publizierte 1853/55 seine Untersuchungen die Mentawej-Inseln und ihre Bewohner betreffend 2, seine Beschreibung von der Insel Engano und deren Bewohner ${ }^{3}$; seine geographische und ethnographische Beschreihung von der Provinz Singkel, der Länder längs des Simpang-kanan gelegen und der Banjak-Inseln, nebst einer kurzen Notiz betreffend den Simpang-kịi ${ }^{4}$. Der Rapport, die Tnsel

${ }^{1}$ Ueber diese Mission nach Neu-Guinea vergl. man J. K. W. Quarles van Ufford, Iets over von Rosenberg's zending naar Nieuw-Guinea: Bijdragen tasl-, Ianden volk. N. I. 3e S. VI, s. 47.

? Bd. I, 1853 , s. $399-440$.

Bd. III, 1855 , s. $370-386$.

4 Bd. III. 1855, s. 397-476. Seine Besohreibung vop einigen Theilen von Seram in Bd. XVI, 1867, s, $97-183$. 
Nias und deren Bewohner betreffend, durch von Rosenberg und J. F. Nieuwenhuizen abgefasst, wurde in die Verhandlungen des ebengenannten Vereins aufgenommen ${ }^{1}$. Die Schwester-Gesellschaft in Batavia, der Königliche Naturwissenschaftliche Verein in Niederländisch Indien, nahm in ihre Zeitschrift nach einander die Beschreibung seiner Reise nach der Südwest- und Nord-Ostkuste von NeuGuinea und nach den Inseln Misool, Salawatti, Batanta und Waigeü auf ${ }^{2}$, nebst einer Reihe naturhistorischer Beiträge, von welchen wir hier seine Uebersichtstabellen für die Ornithologie des Indischen Archipels ${ }^{3}$, den Beitrag zur Ornithologie von Neu-Guinea ${ }^{4}$, den über die Sumatranischen Nashornvögel ${ }^{\mathbf{5}}$, über neue Vogelsorten von Mysool und Salawatti ${ }^{6}$, über einige andere, während seiner Reise nach den Aru- und Kei-Inseln entdeckte Vogelarten ${ }^{7}$, eine Uebersicht der Papageiarten des Indischen Archipels ${ }^{8}$, ein Wort über den grossen Paradiesvogel (Paradisea apoda) ${ }^{\boldsymbol{9}}$, über das Vorkommen von Elephant und Tiger auf Borneo ${ }^{10}$, über den Brunnen Kumaloko in der Minahassa nennen ${ }^{11}$, woran sich andere mehr oder weniger ausgedehnte Aufsätze, kleine Artikel und Recensionen in verschiedenen niederländischen und deutschen Zeitschriften ${ }^{12}$, Tages- und Wochenblättern schliessen.

1 Bd. XXX, 1863, Nr. 1.

2 Vergl. Natuurk. Tijdschr. N. I., XIX, 4e s. V. 1859 , s. $399-422$; XXII, 5е s. II, 1860 , s. $306-353$; XXIV, 5е s. IV, 1862, s. $272,283,333-353.363$, Vergl. Bd. XXIII, 5e s. III, 1861, s. 541. Verg. Miquel, Natuurkundig onderzoek van Ned. Indië: de Gids, Nov. 1864. s. 313.

3 Vergl. Verhandl. Nat. Vereen. V. 1858/59.

4 Nat. Tijdsch r. XXV, 1863, s. 222-256.

a Verh. Nat. Vereen. III. 1857/58.

- Nat. Tijdsohr. XXXIII, 1861 , s. $42-45$.

7 Nat. Tijdsch r. XXIX, 1867, s. 142-145.

8 Nat. Tijdsch r. XXV, 1863, s. $138-148$.

9 Nat. Tijdsch r. XXIX, 1867, s. $136-142$.

10 Nat. Tijdsch r. XIII, 1857 , s. $469-470$.

11 Nat. Tijdschr. XXIX, 1867 , s. $146-148$.

12 Folgende Aufsätze finden wir von ihm aufgenommen besonders in den:

Mittheilungen d. Ornithologischen Vereines zu Wien:

Eine Monstrosität von Fringilla coelebs L.: 8. Jahrg. nr 6.

Nachschrift zu: Eine Monstrosität von Fringilla coelebs: 8. Jahrg. nr 12.

Die Para liesvögel und ihre Verwandten: 9. Jahrg. $\mathrm{n}^{\mathrm{r}}$ 2, 3, 4, 5.

Zu Musschenbroek's Abhandlung, die Paradiesvögel und ihre Verwandten: 9, Jahrg. nr 30.

Zur Biographie der Mainate: 10. Jahrg. nr 13.

Die Zucht des Strausses am Cap der guten Hoffnung; 10. Jahrg. nr 17 . 
Von den drei wichtigen durch von Rosenberg unternommenen Reisen nach den Süd-Oster Inseln, 1865, in der Abtheilung Gorontalo und nach der Geelvink-Bay auf Neu-Guinea, 1869 und 1870 wurden die durch ihn abgestatteten Rapporten nacheinander 1865 , 1867 und 1875 mit einer Anzahl lithographierter und colorierter Bilder und Karten durch das Königliche Institut für die Sprach-, Landes- und Volkskunde Niederländisch Indiens in $3 \mathrm{Bd}$. in 40 . und in $8^{0}$. herausgegeben, denen unser verstorbener Geograf P. J. B. C. Robidé van der Aa jedesmal ein ausführliches und inhaltreiches Vorwort beifügte.

Es war ein glücklicher Gedanke von Rosenberg's den grössten Theil seiner Beiträge, in Zeitschriften und Werken gelehrter Gesellschaften aufgenommen, zusammen $\mathrm{zu}$ tragen und davon eine Bearbeitung in Deutscher Sprache zu besorgen welche 1878 zu Leipzig, Verlag von Gustav Weigel, in drei Abtheilungen

Das. Alter eines Albatros: 11. Jahrg. nr 3.

Zur Lebensbeschreibung des Kuau (Argus giganteus): 11. Jahrg. $\mathrm{n}_{\mathrm{r}} 6$.

Die Falkenbeize bei den Arabern in Algier: 11. Jahrg. nr 4, 5.

Wie alt ein Storch werden kann. Notizen: 11. Jahrg. nr 4.

Abnorme Schnabelbildung bei einem Distelfink: 11. Jahrg. nr 7, 8.

Die Vögel Kamschatka's. Excerpt aus: The Cruise of the Marchesa to Kamschatka and Nieuw Guinea, by F. H. H. Guillemard. London 1866: 11. Jahrg. nr 9, 10.

Dr. E. P. F. Pollen und seine Bedeutung für die Wissenschaft: 11. Jahrg. $\mathrm{n}^{\mathrm{r}} 12$.

Die Vögel von Nord-Borneo. Gesammelt durch F. H. H. Guillemard. Excerpt aus the Cruise of the Marchesa to Kamschatka and New-Guinea: 11. Jahrg. $\mathrm{n}_{\mathrm{r}} 11$.

Vögel von Sumbawa u. Selebes. Gesammelt durch F. H. H. Guillemard. Excerpt aus: The Cruise of the Marchesa etc. etc.: 11. Jahrg. nr 12.

Nachträgliches zu den Vögeln Kamschatka's, von Dr. Guillemard: 12 Jahrg. n $\mathrm{n}^{\mathrm{r}} 5$.

Vögel von den Molukken, Neu Guinea und umliegenden Inseln. Excerpt aus The Cruise etc. : 12. Jahrg. n 9 .

Das Sandhuhn in Holland. Notizen: 12. Jahrg. nr 10.

Die Vertheilung der Psittaciden über die Inseln des Ostindischen Archipels: Journ. Ornithol. X, 1862 , s. 59-68.

Ein Wort über Casuarius Kaupi: Journ. Ornithol. XXI, 1873, s. 390.

Berichtigung zu dem Artikel : "Das Reichsmuseum in Leyden, von F. von Drosten": Journ. Ornithol. XXI, 1873 , s. 391.

Verbreitung der Paradiesvogel: Frankfurt. Zool. Garten. XIV, 1873, s. 195. Die Erddrosseln (Pitta) von Insulinde: Frankfurt. Zool. Garten. XIX, nr 11. Ein Erlebniss mit dem Tiger auf Java: Frankfurt. Zool. Garten. XX, nr 10. Vögel von Neu Guinea's Südküste: Frankfurt. Zool. Garten. XXII, nr 1. Ein Jäger Eldorado: Frankfurt. Zool. Garten. XXII, nr 6.

Die Affen von Insulinde: Frankfurt. Zuol. Garten. XXIII, nr 4.

Unser Maulwurf: Darmstädter Thierschutz-Ver. II. Jahrg. $\mathrm{n}_{\mathrm{r}} 1$.

Les Hattąms (Nouvelle-Guinée): Annales de l'extrême Orient. I, 1879, s. 219. 
in 80. herausgegeben wurde, unter dem Titel: "Der Malayische Archipel. Land und Leute in Schilderungen, gesammelt während eines dreissigjährigen Aufenthaltes in den Kolonien", von welcher Arbeit Thre Majestät die Königin der Niederlande, Emma, gnädigst geruhte die Dedikation anzunehmen. Dieses Werk verdient auch besonders Verıneldung der vielen schönen Holzschnitte wegen, die demselben zur Zierde gereichen, nur ausnahmsweise schon früher herausgegebene Bilder wiederholen und in jedem Falle durch künstlerischen Werth die lithographischen Abbildungen übertreffen, die den originalen Ausgaben der einzelnen Reiseberichte bisweilen beigegeben wurden. Uebrigens enthält diese Ausgabe, wie mit Recht im Vorwort, womit Professor P. J. Veth diese Schrift bei dem Publikum einführte, bemerkt wird, Nichts was für uns Niederländer nicht seit längerer oder kürzerer Zeit zugänglig war; jedoch es freut uns, dass R. jetzt durch diese verkürzte Gesammtausgabe seiner Werke in seiner Muttersprache zugleich dem Interesse der Wissenschaft in grösserem Kreise diente, sich dadurch selbst die Ehrenstelle vindicirte, die ihm unter den Erforschern des Malayischen Archipels

Les flles Kei. Notes ethnographiques: Annales de l'extrême Orient. 1880, nr. 20 , s. 231 .

Moeurs et coutumes des Arfaks. Avec carte: Annales de l'extrême Orient, 1878, nr. 1 .

La grande baie du Geelvink (Nouvelle-Guinée): An nales de l'extrême Orient, I, 1878 , s. 193

De paradijsvogel $(1760-1860-1870)$. - De reuzen-alk. Met eene plaat: J a arboekjevan het Kon. Zool. Gen. Natura Artis Magistra, 1873, s. 169; 1874 , s. 144 .

Benoodigheden van den passagier naar en van de Koloniën, den pionier-kolonist en den wetenschappelijken reiziger: Catalogus der afd. Nederl. koloniën van de Intern. koloniale Tentoonstelling te Amsterdam, 1883. Leiden 1883 , III, s, 224-223.

De Papoea's van Nieuw-Guinea: Bijdragen Kon. Instituut t. d. taal-, land-en volkenk. N. I., 3e s. X. s. 386 , XI, s. 392 .

Eenige aanteekeningen naar aanleiding van het Dagboek van Bernstein's laatste reis, bewerkt door Mr. S. C. J. N. van Musschenbroek: Bijdragen Kon. Instituut t. d. taal-, land-en volkenk. N. I. 4e s. VIII. s. 119-122.

Korte beschrijving der Teloeti-baai. Met een kart: Tijdschr. Nederl. Aardr. Genootsch., II, no. 3 , s. $147-150 ; n^{0} .9$.

Nieuw Brittanje geschetst in losse tafereelen van land en volk. Naar het En gelsch van Powel: Tijdsehr. Nederl. Aardr. Genootsch., 2e s. III, IV, Afd. Verslagen en A ard." Meded.

De kleine Oost. Excerpten uit een reisjournaal: Tijdschr. Nederl. A ardr. Genootseh. 2es. IV. Afd. Verslagen en Aard. Meded.

Recensionen folgender Schriften, aufgenommen in der Zeitschrift In dis che Gids ;

J. Lorié, Over de Javaansche Eruptief-gesteenten, II Jahrg. I. s. 292. 
gebührt, und der Regierung, deren treu€r, eifriger, kenntnissreicher und wahrheitliebender Diener er seit vielen Jahren gewesen, das Lob spendete, das sie beanspruchen kann.

Mit Rücksicht auf von Rosenberg's fruchtbare Arbeit kann es nicht wundern dass Fürsten und wissenschaftliche Anstalten ihn um die Wette ehrten. Im April 1861 wurde ihm der Haus- und Verdienst-Orden Philipp des Grossmüthigen von Hessen geschenkt, im September und November 1864 der Grossherzoglich Hessische Ludwigs Orden $1^{\mathbf{r}}$ Klasse und der Orden des Zähringer Löwen von Baden, im März und April 1867 die goldene Verdienst-Medaille für Wissenschaft und Kunst vom Gross-Herzogthum Hessen, die 2e Klasse des Herz. Nassau-Ernest. Sächsischen Haus-Ordens und der Würtembergische Kronen-Orden. Mit Königlichem Beschluss vom 14 Juli $1878 \mathrm{n}$ r. 39 wurde ihm von der Niederländischen Regierung die Silberne Medaille zuerkannt wegen des von ihm an den Tag gelegten Interesses an den Reichs-Sammlungen für Wissenschaft und Kunst, in Gestalt werthvoller Geschenke an das Ethnographische Reichs Museum und das Naturhistorische Museum zu Leiden. Von Rosenberg's Sammlungen von Nord Selebes und den Aru- und Kei-Inseln sind auch heut noch fast die einzigen Vertreter jener Kulturen im Ethnographischen Reichs Museum.

Die Bataviasche Gesellschaft der Künste und Wissenschaften

0. Sachot, Récits de voyage. Nègres et Papous, II Jahrg. I. s. 940.

Stone, A few months in New Guinea, II Jahrg. II. 8. 145.

K. Martin, Die Tertiärschichten auf Java. III Jahrg. I. s. 1001.

J. H. van Balen, Avontuurlijke reisen. III. De roode paradijsvogel. Eene handelsreis naar Ned. Nieuw-Guinea. III Jahrg. II. s. 997.

M. T. H. Perelaer, Borneo van Zuid naar Noord. III Jahrg. I. Vergl. M. T. H. Perelaer, Mijn antwoord aan den heer H. von Rosenberg. IV Jahrg. II. 8. 198 u. H. von Rosenberg, Een laatste woord omtrent Perelaer's Borneo van Zuid naar Noord. IV Jahrg. II. s. 203.

Sammlungen des geol. Reichsmuseums in Leiden. V Jahrg. II. 8. 84; VI J. II s. 182 ; VII J. I. s. 509 ; VIII J. I. s. 245 ; IX J. I. s. 425 , II, 8. 1159 , 1504, 1910 Th. Posewitz, Unsere geologischen Kenntnisse von Borneo. V Jahrg. II, 8. 84.

Th. Posewitz, Die Zinninseln im Indisohen Oceane. VII Jahrg. II.s. 907 ; VIII J. II. s. 1082 .

0. Mohnike, Blieke auf das Pflanzen- and Thierleben in den Nied. Malaienländern: V Jahrg. II. 8. 335

A. Pagenstecher, Beiträge zur Lepidopteren-Fauna von Amboina. VII Jahrg. I. 8. 508; VIII J. I. s. 538 ; IX J. I. 8. 40.

O. Staudinger, Exotische Schmetterlinge. VII Jahrg. II. 8. 1810; VIII J. I. s, 245 ; IX Jahrg. I. s. 41.

A. Haga, Nederlandsch Nieuw-Guinea. VII Jahrg. I. s. 669. 
emannte ihn im Juli 1857 zum Mitglied, wie dies schon im December 1856 der Königl. Naturwissenschaftliche Verein in Niederl. Indien that; und als die Indische Geographische Gesellschaft ihn am 5 September $1860 \mathrm{zum}$ correspondirenden Mitglied ernannte, beehrten ihn die Niederl. Geogr. Gesellschaft zu Amsterdam, 22 December 1877, und die Königl. Zoöl. Gesellschaft Natura Artis Magistra, 8 Januar 1872 mit der Ehren Mitgliedswürde. Unser Königl. Institut für die Sprach-, Landes- und Volkskunde Niederl. Indiens zählte ihn seit Januar 1872 zu seinen Mitgliedern und verlieh ihm im April 1878 den Titel eines eorrespondirenden Mitgliedes. Schliesslich ehrte ihn auch sowohl der Ornithologische Verein zu Wien, als die Kaiserlich-LeopoldinischCarolinische deutsche Akademie der Naturforscher zu Halle a/S. durch Ernennung zu ihrem Mitgliede.

Bei Gelegenheit des Internationalen Congresses und der Exposition für Geographie, zu Paris 1875, ehrte die Internationale Jury auf besonders schmeichelhafte Weise seine, durch des Königl. Institut publizierte Ausgaben 1.

Nacî seriner Räiktuble ous Indian lings B. sich erst in Darmstadt nieder, und seit 1875 lebte er ununterbrochen im Haag. Eine treue und sorgliche Gattin, Freiin Caroline Elisabeth Louise von Breidenbach zu Breidenstein aus Darmstadt - mit welcher er sich vor seiner Abreise aus Europa (23 April 1867) vermählt hatte erhöhte sein Lebensglück und blieb Zeuge der wissenschaftlichen Arbeit welcher er sich ohne Unterlass widmete, bis er, nach

${ }^{1}$ Der Vorstand des Königl. Institut für die Sprach-, Landes- und Volkskunde v. N. I. hatte aus Paris, am 11. Aug. 1875 den folgenden "Lettre de distinction" empfangen:

"L'Exposition de l'Institut Royal des Рays-Bas a paru au_Jury_International mériter une récompense exceptionnelle.

"Les études si remarquables de cet établissement sur la géographie, l'ethogra-

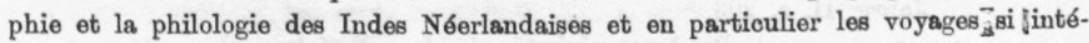
ressants de Rosenberg, présentent un tel intérêt scientifique, que les distinctions prévues par le réglement ne pouvaient leur être appliquées.

"J'ai l'honneur, au nom du Congrès, de porter à votre connaissance cette haute appréciation de Jury et de vons délivrer, pour l'Institut Royal des Pays-Bas la présente_lettre de distinction, comme la récompense de l'ordre le plus élevé decernée à l'oceasion de l'exposition.

"Veuillez agréer, Monsieur le Président, l'assurance de ma haute considération.

Le Vice-Amiral, Président du Congrès et? de la Societe de Geographie de Paris.

(gez.) Dr LA Ronctìre LE Noury.

Vergl. Bijdragen Kon. Instituut t. d. taal-, land-en volkenk. v. N.I. 3e s. XI, s. XXXV. 
dreiwöchentlichem Krankenlager, am Abend des 15 November d. J. ron ihrer Seite gerissen wurde. Seinem Wunsche zufolge wurden seine irdischen Ueberreste in der Familiengruft zu Darmstadt beigesetzt.

Noch drei Wochen vorher hatte unser Mitglied des Kon. Instituts, Herr Schmeltz, die Freude von Rosenberg zu sprechen; er war gekommen um mit ihm Verabredungen wegen Publikationen im "Internationalen Archiv für Ethnographie" festzustellen, für welches er eben erst seinen ersten Aufsatz: "Een en ander over de bewoners van de Mentawei-Eilanden" eingesandt hatte, der im 6 ten Heft dieses Jahrgangs abgedruckt ist. Voll war er noch der Pläne für die Zukunft und sein unerwartetes Hinscheiden trifft auch das Archiv sehr hart.

Für den grösseren Theil seiner Stadtgenossen und für die jüngeren unter , unsern Jandsleuten war von Rosenberg ein Fremder geworden, aber in den literarischen und wissenschaftlichen Kreisen, besonders in denjenigen, deren Hauptwirksamkeit in der Verbreitung und Beförderung der Kenntniss der Geographie und Ethnographie unserer schönen Kolonien liegt, blieb er als ein alter Bekannter und Freund geehrt und geachtet; durch seinen Heimgang verlieren sie einen der talentvollen Mitarbeiter und Mitstreiter, dessen unermüdete Wirksamkeit im Lande der Tropen, während eines dreissigjährigen Aufenthaltes, Ehrfurcht und Dankbarkeit einflösst und ihm eine Ehrenstelle sichert unter den verdienstvollen Indologen und Naturforschern des letzten halben Tahrhunderts. Schreiber dieses, der den Vorzug hatte, während vieler Jahre zu von Rosenberg's Freunden gezählt zu werden, war es ein Bedürfniss seinem Gedächtniss ein Wort zu widmen im Organ einer Stiftung, deren Schriftenreihe durch seine werthvollen Beiträge vermehrt wurde und fïr deren Wirken von Rosenberg stets warme Sympathie zeigte. 